\title{
A Constrained Resource Allocation Study for LOS MIMO Fading Ad-Hoc Networks
}

\author{
Homayoun Yousefi'zadeh \\ Department of EECS \\ University of California, Irvine \\ hyousefi@uci.edu
}

\begin{abstract}
We study constrained resource allocation problems for MIMO wireless ad-hoc networks under LOS fading. By considering the tradeoff between the aggregate consumed power and throughput of such networks, we investigate the performance characteristics of a number of MIMO coding techniques, namely MRC, STBCs, and orthogonal STTCs. Our study shows that utilizing space-time coding techniques can decrease the power consumption and/or increase the achievable throughput of ad-hoc networks under loss constraints.
\end{abstract}

Index Terms-Power, Throughput, Ad-Hoc Networks, Rician Fading, Markov Chain, MIMO.

\section{INTRODUCTION}

Addressing the tradeoff between the achievable throughput as a metric of Quality of Service (QoS) and the consumed power under the presence of fading is arguably an important issue in ad-hoc networks. The optimization of wireless spectrum has been the focus of research efforts as it pertains to power, bandwidth, and received signal-to-interference-noise ratio. Andersin et al. [2], Bambos et al. [3], and Ulukus et al. [14] proposed various iterative methods to maximize the minimum signal-to-interference ratio $(S I R)$, to minimize total or individual power, or to maximize throughput under some kind of QoS constraint. Relying on Geometric Programming (GP), Chiang et al. [4] solved a set of resource allocation problems for QoS provisioning in wireless ad-hoc networks. Hayajneh et al. [6] proposed a game-theoretic power control algorithm for wireless channels. Shah et al. [9] and Ramanathan et al. [8] investigated the issue of power control in wireless networks with the considerations of network topology.

The material presented in this paper addresses some important open areas left out in our earlier works of [15] and [16]. In those articles, the temporally correlated loss behavior observed in a wireless channel was modeled with a finite-state Markov chain extracted from the distribution of Rayleigh fading channel. representing a rich scattering NLOS environment. In order to compensate for the correlated loss effects of the fading channel, we proposed the use of Reed-Solomon (RS) channel coders. Relying on an analysis of channel coding, we focused on a pair of centralized resource allocation problems subject to power or data rate ${ }^{1}$ along with loss constraints. The studies of [15] and [16] compared the performance of Maximum Ratio Combining (MRC) and Space-Time Block Codes (STBCs) techniques in rich scattering ad-hoc topologies operating over short distances. However, performance gains of such resource

\footnotetext{
This work was sponsored by Boeing Integrated Defense Systems.

${ }^{1}$ In this paper, the terms data rate and bandwidth are used interchangeably. They should not be confused with the term channel coding rate.
}

allocation schemes under LOS fading and utilizing more sophisticated space-time coding techniques were not investigated in our previous works. Such an investigation is of importance due to the significant departure from the underlying assumptions related to path gains and broadband data rates. The contributions of this work are in the following areas. First, we analyze the effects of operating under LOS Rician fading. Second, we investigate the accommodation of mobile nodes utilizing orthogonal Space-Time Trellis Codes (STTCs) in addition to MRC and STBCs. Relying on our general model, we formulate and solve a pair of constrained resource allocation problems. We also validate our results under mobility applied to longer ranges of operation that are typical for LOS links.

The rest of this paper is organized as follows. Section II revolves around the underlying system analysis. In Section III, we formulate and solve a pair of optimization problems. The first problem is a power optimization problem aimed at minimizing total transmission power subject to block loss probability, aggregate data rate, and maximum available per link powers. The second problem is a throughput maximization problem subject to consumed powers and similar other constraints as the first problem. Section IV provides numerical results of our experiments validating our analysis under node mobility. Finally, Section V concludes this work.

\section{System ANALYSIS}

In this section, we provide an analysis of the underlying communication system based on the characteristics of the fading wireless channel. The following subsections include an analysis of the instantaneous signal-to-interference-noise ratio $(S I N R)$ in the fading channel, a discussion of channel modeling with finite-state Markov chains, calculation of symbol error rates over the links associated with space-time coded mobile nodes, and the channel coding scheme proposed for compensating the effects of correlated loss observed over wireless links.

\section{A. Analysis of Received $S I N R$}

Consider $n$ wireless links, labeled $\mathcal{L}_{1}, \ldots, \mathcal{L}_{n}$, on which transmission powers are $P_{1}, \ldots, P_{n}$, respectively. Link $i$ is associated with the $i$-th transmitter/receiver pair. Each link may be connecting multiple antenna mobile nodes. Suppose, per symbol transmission power $P_{j}$ is equally distributed among $M_{j}$ transmit antennas of link $j$. The number of receive antennas for link $j$ is assumed to be $N_{j}$. The nonnegative number $G_{i j}(t)$ represents the path gain in the absence of fading from the transmitter of link $j$ to the receiver of link $i$ at time $t . G_{i j}(t)$ captures such factors as path loss, shadowing, antenna gain, and so 
on. We denote $F_{i j}^{(m, n)}(t)$ as the fading factor between transmitting antenna $m$ of link $j$ and receiving antenna $n$ of link $i$. Then, $S I N R$ at time $t$ for link $i$ determines the quality of the received signal. Treating the paths between different pairs of transmit and receive antennas as independent ones, the instantaneous $S I N R$ is defined as

$$
S I N R_{i}(t)=\frac{G_{i i}(t) \frac{P_{i}(t)}{M_{i}} \sum_{m=1}^{M_{i}} \sum_{n=1}^{N_{i}} F_{i i}^{(m, n)}(t)}{\sum_{j \neq i} G_{i j}(t) \frac{P_{j}(t)}{M_{j}} \sum_{m=1}^{M_{j}} \sum_{n=1}^{N_{i}} F_{i j}^{(m, n)}(t)+N_{i} n_{i}(t)}
$$

noting that the power of white Gaussian noise signal $n_{i}(t)$ on link $i$ is multiplied by $N_{i}$. Considering the magnitudes of the base and interference signals and the sources of the signals, we note that the fading factors between a transmitter and a receiver on different links are identically and independently distributed (i.i.d). However, this assumption is not necessarily true when the transmitter and receiver belong to the same link. Generally speaking, the latter represents a temporally correlated channel in which the correlation between $F_{i j}(t)$ and $F_{i j}(t+\Delta t)$, where $\Delta t$ is a given time shift, cannot be ignored. In order to capture temporal correlation of the wireless channel, we make few realistic assumptions as follows. First, we assume that the fading factors in interfering signals $F_{i j}$ 's where $i \neq j$, are i.i.d. We note that the latter assumption does not lead to a loss of generality as it may be relaxed relying on a similar discussion to the one furnished for the fading components $F_{i i}$. Second, $F_{i j}$ 's have unit means so long as $G_{i j}$ 's are appropriately scaled to reflect variations from this assumption. Third, the noise $n_{i}(t)$ has a mean of $n_{i}$. Fourth, when the wireless channel varies slowly with respect to symbol interval, $P_{i}(t)$ and $G_{i j}(t)$ can be viewed as constants and $F_{i j}(t)$ as a random variable within the symbol interval. Based on the above assumptions, we treat the average signal-to-interference-noise ratio for link $i$ as a random variable in the form of

$$
\begin{aligned}
S I N R_{i} & =\frac{E\left[G_{i i}(t) \frac{P_{i}(t)}{M_{i}} \sum_{m=1}^{M_{i}} \sum_{n=1}^{N_{i}} F_{i i}^{(m, n)}(t)\right]}{E\left[\sum_{j \neq i} G_{i j}(t) \frac{P_{j}(t)}{M_{j}} \sum_{m=1}^{M_{j}} \sum_{n=1}^{N_{i}} F_{i j}^{(m, n)}(t)+N_{i} n_{i}(t)\right]} \\
& =\frac{G_{i i} \frac{P_{i}}{M_{i}} \sum_{m=1}^{M_{i}} \sum_{n=1}^{N_{i}} F_{i i}^{(m, n)}}{\sum_{j \neq i} N_{i} G_{i j} P_{j}+N_{i} n_{i}}
\end{aligned}
$$

We note that in the right hand side of the equation above $F_{i i}^{(m, n)}$ represents a random variable and the rest of the terms are constants. Hence, it suffices to examine the distribution of $F_{i i}^{(m, n)}$ in order to obtain fading statistics of $S I N R_{i}$. We start our discussion from the case of a single transmit and single receive antenna link, i.e., $F_{i i}=F_{i i}^{(1,1)}$ and $M_{i}=N_{i}=1$. We refer to such a case as a $1 \times 1$ link case. For an LOS environment, we rely on the Rician model with the fading factor $\alpha$ to relate the input and output of the channel. The quantity $y=|\alpha|$ has a marginal Rician probability density function (pdf) in the form of

$$
p_{Y}(y)=\frac{y}{\sigma^{2}} \exp \left(-\frac{y^{2}+\mu^{2}}{2 \sigma^{2}}\right) I_{0}\left(\frac{y \mu}{\sigma^{2}}\right), y \geq 0
$$

where $\mu>0$ is the amplitude of the LOS signal, $\sigma^{2}$ is half of the average power of all of the multipath components, and $I_{0}$ is the zero-order modified Bessel function of the first kind given by

$$
I_{0}(z)=\sum_{l=0}^{\infty} \frac{(z / 2)^{2 l}}{(l !)^{2}}
$$

with $z \geq 0$. Considering the fact that $F_{i i}$ is defined as $F_{i i}=$ $y_{i}^{2}=\left|\alpha_{i}\right|^{2}$, we are interested in the distribution of $\left|\alpha_{i}\right|^{2}$. We note the following properties of random variables [7].

Property 1: If the random variable $y_{1}$ has a pdf in the form of $f_{Y_{1}}\left(y_{1}\right)$, then the random variable $y_{2}=y_{1}^{2}$ has a pdf as

$$
f_{Y_{2}}\left(y_{2}\right)=\frac{1}{2 \sqrt{y_{2}}} f_{Y_{1}}\left(\sqrt{y_{2}}\right)
$$

Utilizing Equation (3), we conclude that $F_{i i}$ has a marginal pdf as shown in Equation (5).

$$
p_{F}\left(F_{i i}\right)=\frac{1}{2 \sigma^{2}} \exp \left(-\frac{F_{i i}+\mu^{2}}{2 \sigma^{2}}\right) I_{0}\left(\frac{\mu \sqrt{F_{i i}}}{\sigma^{2}}\right), \quad F_{i i} \geq 0
$$

In [15], we provide similar expressions for NLOS environments utilizing a Rayleigh fading distribution.

Next, we apply our discussion to the case of a link with $M_{i}$ transmit and $N_{i}$ receive antennas. Given the joint pdf of the random variables $F_{i i}^{(m, n)}$ and under the assumption of spatial independence, the joint pdf of fading on an $M_{i} \times N_{i}$ link can be specified relying on the following property of random variables [7].

Property 2: If the pdf set $\left\{f_{Y_{1}}\left(y_{1}\right), \cdots, f_{Y_{q}}\left(y_{q}\right)\right\}$ is associated with the set of independent random variables $\left\{y_{1}, \cdots, y_{q}\right\}$, then the pdf of their sum $z=y_{1}+\cdots+y_{q}$ is calculated as $f_{Z}(z)=$ $f_{Y_{1}}(z) * \cdots * f_{Y_{q}}(z)$ where $*$ represents the convolution operator. It is important to note that the number $q$ represents the product of $M_{i} \times N_{i}$ in the case of a link with $M_{i}$ transmit and $N_{i}$ receive antennas.

Having specified the probability density functions, we can now focus on capturing temporally correlated loss of Rician fading channels. We propose partitioning and modeling such an analog channel with a digital Markov chain model. A Markov chain is fully specified by a set of state transition probabilities and per state error probabilities. While the transition probabilities are calculated based on the average received $S I N R$, per state error probabilities are expressed in terms of modulation and space-time coding characteristics in addition to $S I N R$.

We apply the fundamental idea of partitioning to the pdf of $F_{i i}$ in order to model the underlying Rician fading channel with a finite-state Markov chain. In [15], we discuss the details of partitioning the pdf of a random variable.

Similar to [16], this study relies on average natural burst lengths to specify the set of state transition probabilities in a Gilbert-Elliott Markov chain. We propose choosing a set of partitioning thresholds $\left\{\xi_{0}=0, \xi_{1}, \xi_{2}=I N F\right\}$ such that

$$
\int_{\xi_{s-1}}^{\xi_{s}} p_{F}\left(F_{i i}\right) d F_{i i}=s_{s s}, \quad s=1, \ldots, S
$$

where $s_{s s}$ represents the steady state probability of being in state $s$. Once the thresholds are set, they allow us to find $F_{i i, s}$, the representative value of the conditional pdf of $F_{i i}$ in state $s$, by

$$
F_{i i, s}=\int_{\xi_{s-1}}^{\xi_{s}} F_{i i} p_{F}\left(F_{i i}\right) d F_{i i}, \quad s=1, \ldots, S
$$


Therefore, the corresponding representative values of $S I N R_{i}$ 's are given by

$$
S I N R_{i, s}=\frac{G_{i i} P_{i} F_{i i, s}}{\sum_{j \neq i} G_{i j} P_{j}+N_{i} n_{i}}, \quad s=1, \ldots, S
$$

The temporal dynamics of the Markov chain are determined by a matrix of one-step-transition probabilities $\Pi_{S \times S}$. Given the steady state probabilities of being in state $s$ for $s \in\{1, \cdots, S\}$ and the average natural burst lengths, matrix $\Pi$ can be specified. Due to the finite value of the error introduced by the model and the transitioning assumption, we must modify the transition matrix such that each row adds up to one. This can be done by uniformly scaling each row by its sum as $\pi_{s r}=\tilde{\pi}_{s r} /\left(\sum_{s=1}^{S} \tilde{\pi}_{s r}\right)$. We will explain the details of the Markov chain dynamics in Section II-C.

\section{B. Calculation of MIMO Symbol Error Rates}

In this section, we calculate the symbol error rate of a MIMO link under the assumption of a facing fading Rician (LOS) channel and utilizing PSK modulation. We also note that our approach can be applied to other modulation schemes such as QAM.

First, we introduce the symbol error rate of a fast Rician fading link associated with single transmit and $N$ receive antenna mobile nodes using maximum ratio combining (MRC). Starting from Equation (9.15) of [10], the symbol error rate of a single transmit $N$ receive antenna system using maximum ratio combining (MRC) can be calculated as

$$
\begin{aligned}
S E R=\frac{1}{\pi} & \int_{0}^{(L-1) \pi / L}\left[\frac{(1+\kappa) \sin ^{2} \phi}{\left.\overline{S I N R} \sin ^{2}\left(\frac{\pi}{L}\right)+(1+\kappa) \sin ^{2} \phi\right)}\right. \\
& \left.\times \exp \left(-\frac{\kappa \overline{S I N R} \sin ^{2}\left(\frac{\pi}{L}\right)}{\overline{S I N R} \sin ^{2}\left(\frac{\pi}{L}\right)+\left(1+\kappa \sin ^{2} \phi\right)}\right)\right]^{N} d \phi
\end{aligned}
$$

where $\kappa=\frac{\mu^{2}}{2 \sigma^{2}}$ represents the Rician fading parameter. While there is no closed-form solution to the integral above, its value can be numerically evaluated. We note that the integral is reduced to the case of Rayleigh fading for NLOS fading environments with $\kappa=0$. From Equation (9), one can calculate the symbol error rate of a link associated with single transmit single receive antenna nodes as well as a link associated with single transmit double receive antenna nodes by setting $N$ to 1 and 2, respectively. Relying on a discussion of diversity gains, we also argue that the symbol error rate of the space-time block codes (STBCs) of [1] and [13] can be calculated from Equation (9) by proper mapping of the values of $S I N R$.

For example, the symbol error rate of a $2 \times 1$ Rician link can be calculated by by setting $N$ to 2 in Equation (9) and using appropriate $S I N R$ from the counter part of Equation (8) in the case of $2 \times 1$ antenna configuration.

Calculating symbol error rates of orthogonal STTCs is more complicated than those of STBCs. Based on the work of Simon et al. [11], the symbol error rate of a $2 \times N$ link utilizing orthogonal STTCs and under fast Rician fading is given by

$$
\begin{aligned}
S E R=\frac{1}{\pi} & \int_{0}^{\pi / 2}\left[\prod_{p=1}^{P P}\left(\frac{(1+\kappa) \sin ^{2} \phi}{\overline{S I N R} \frac{d_{p}^{2}}{4}+(1+\kappa) \sin ^{2} \phi}\right)\right. \\
& \left.\times \exp \left(-\frac{\kappa \overline{S I N R} \frac{d_{p}^{2}}{4}}{\overline{S I N R} \frac{d_{p}^{2}}{4}+(1+\kappa) \sin ^{2} \phi}\right)\right]^{2 N} d \phi
\end{aligned}
$$

where $P P$ is the number of observed symbols per transmit antenna and $d_{p}^{2}$ denotes the squared Euclidean distance between the transmitted and chosen pair of transmitted symbols in the $n$-th symbol interval. We also note that according to [11], the choice of the Trellis of Fig. 1 along with $P P=1$ yields $d_{p}^{2}=8$.

0

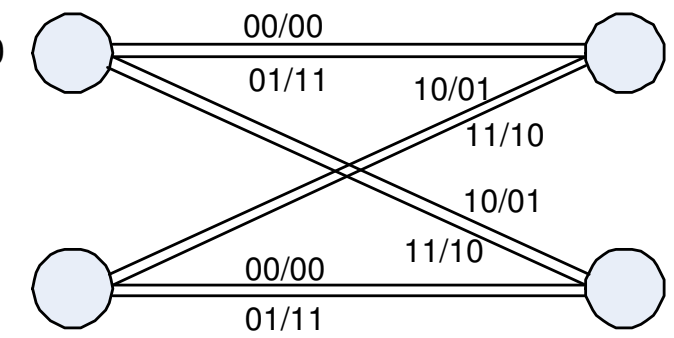

Fig. 1. Trellis diagram for rate 1, 2-state BPSK orthogonal STTC.

\section{Proposed Channel Coding Scheme}

We propose the use of Reed-Solomon (RS) channel coders to compensate for temporally correlated loss effects of the wireless channel. An RS channel coder $R S(b, k)$ converts $k$ symbols into a $b$-symbol block by appending $(b-k)$ parity symbols. Such a channel coder introduces a channel code rate of $r=\frac{k}{b}$ and is able to correct as many as $t_{C}=\left\lfloor\frac{b-k}{2}\right\rfloor$ symbol errors in a block. Let $\varphi(b, k, G)$ and $\varphi(b, k, B)$ denote the probability of receiving exactly $k$ symbols from $b$ symbols and winding up in the GOOD and the BAD state of the Gilbert-Elliott model, respectively. The probability of receiving exactly $k$ symbols from a $b$-symbol block for the Gilbert-Elliott model is given by

$$
\varphi(b, k)=\varphi(b, k, G)+\varphi(b, k, B)
$$

The recursive probabilities of receiving exactly $k$ symbols from $b$ transmitted symbols and winding up in the GOOD state and the BAD state are respectively given [15] by

$$
\begin{aligned}
& \varphi(b, k, G)= \\
& S E R_{G}[\gamma \varphi(b-1, k, G)+(1-\beta) \varphi(b-1, k, B)] \\
& \left(1-S E R_{G}\right)[\gamma \varphi(b-1, k-1, G) \\
& +(1-\beta) \varphi(b-1, k-1, B)] \\
& \varphi(b, k, B)= \\
& S E R_{B}[(1-\gamma) \varphi(b-1, k, G)+\beta \varphi(b-1, k, B)] \\
& \left(1-S E R_{B}\right)[(1-\gamma) \varphi(b-1, k-1, G) \\
& +\beta \varphi(b-1, k-1, B)]
\end{aligned}
$$

for $b \geq k>0$, steady state probability of the GOOD state $g_{s s}=\frac{1-\beta}{2-\gamma-\beta}$, steady state probability of the BAD state $b_{s s}=$ $\frac{1-\gamma}{2-\gamma-\beta}$, and the initial conditions

$$
\begin{aligned}
\varphi(0,0, G) & =g_{s s}=\frac{1-\beta}{2-\gamma-\beta} \\
\varphi(0,0, B) & =b_{s s}=\frac{1-\gamma}{2-\gamma-\beta} \\
\varphi(1,0, G) & =S E R_{G}\left[\gamma g_{s s}+(1-\beta) b_{s s}\right] \\
\varphi(1,0, B) & =S E R_{B}\left[(1-\gamma) g_{s s}+\beta b_{s s}\right]
\end{aligned}
$$


If at least $b-t_{C}$ symbols are correctly received from $b$ transmitted symbols, the whole block is recoverable. Hence, the block-loss probability is expressed as

$$
\Psi=1-\sum_{k=b-t_{C}}^{b} \varphi(b, k)
$$

\section{Resource Allocation Problems}

In this section, we formulate a pair of constrained resource allocation problems and also provide their solutions.

Unlike the work of [15] that relies on an adaptive modulation and a fixed channel coding scheme, we propose the use of a fixed modulation and an adaptive channel coding scheme in this work. As such, the set of per link data rates $R_{i}$ are defined as

$$
R_{i}=\epsilon \frac{r_{i}}{T} \log _{2} L_{i}
$$

where $r_{i}$ is the channel coding rate, $\frac{1}{T}$ is the baseband bandwidth, $\epsilon$ is the bandwidth translation factor that captures transmission energy per bit, and $L_{i}$ is the constellation size.

\section{A. Throughput Maximization}

Our objective is to maximize the overall system throughput under the constraints related to per link block-loss probabilities, minimum link data rates, and powers. As an alternative, we also seek to minimize the power of transmission under the constraints related to throughputs, minimum link data rates, and per link block-loss probabilities. The throughput maximization problem appears in the following form.

$$
\begin{array}{cl}
\max _{P_{i}, r_{i}} & R_{\text {total }}=\sum_{i=1}^{n} R_{i} \\
\text { Subject To }: & \Psi_{i} \leq \Psi_{i, u b}, \quad \forall i \\
& R_{i, l b} \leq R_{i}, \quad \forall i \\
& P_{i, l b} \leq P_{i} \leq P_{i, u b}, \quad \forall i
\end{array}
$$

We note that in the formulation above, $P_{i}$ 's and $r_{i}$ 's are the decision variables. Further, we note that the $S E R$ parameters of the Markov chain discussion of Section II-C appear in the form of a set of per link parameters.

Expressions (2), (9), and (16) show that the data rates $R_{i}$ 's are related with per link channel coding rates. Therefore, the objective function (17) is to be optimized over all feasible powers and constellation sizes. The set of constraints represent the maximum allowable block-loss probability on each link, a minimum per link guaranteed throughput, and regulatory or system limitations on transmission powers.

A discussion of solving the optimization problem formulated by (17) along with the constraint sets (18) is provided in [15]. The solution utilizes SQP and a variant of the quasi-Newton method.

We end this subsection by presenting an analysis of the computational complexity for the approach described above. The time complexity of solving the constrained problem of (17) without the discrete constraints associated with the channel coding values of the RS coder is $\mathcal{O}(I d \log d)$ where $I$ indicates the number of iterations and $d$ indicates the degree of the quadratic estimation. For moderate values of $I$, the complexity results are quite good compared to other recursive optimization approaches such as dynamic programming introducing a time complexity in the order of $\mathcal{O}\left(d^{2}\right)$. In order to accommodate the discrete channel coding constraints, we evaluate the objective function at the lower and upper integer bounds of the optimal channel coding rate. By selecting the bound introducing a larger objective function, we have experimentally observed that the sub-optimality of our estimation is quite acceptable in comparison to solving a discrete nonlinear optimization problem introducing a much higher complexity.

\section{B. Power Minimization}

The discussion of this subsection revolves around formulating the power minimization problem and providing a solution to it. We minimize the sum of per link transmission powers subject to block-loss probability requirements, aggregate data rate, and maximum per link available powers. Our optimization problem is formulated as

$$
\begin{array}{cl}
\min _{P_{i}, r_{i}} & P_{\text {total }}=\sum_{i=1}^{n} P_{i} \\
\text { SubjectTo: } & \Psi_{i} \leq \Psi_{i, u b}, \quad \forall i \\
& R_{i, l b} \leq R_{i}, \quad \forall i \\
& P_{i, l b} \leq P_{i} \leq P_{i, u b}, \quad \forall i
\end{array}
$$

where per link transmission powers $P_{i}$ 's and channel coding rates $r_{i}$ 's are the decision variables. The objective function (19) is to be optimized over all feasible per link powers and constellation sizes. The three sets of constraints are enforced to assure maximum block loss probability of the channel coder, minimum link data rate, and maximum available system powers. Similar to the throughput maximization problem, the power minimization problem can be solved relying on SQP and a variant of the quasi-Newton method with the same complexity. The same estimation approach can be used to accommodate discrete channel coding constraints.

We note that the formulations of both problems can be applied either to an overall topology or on a per node basis with a sub-topology of links attached to the node. While the former case represents a centralized problem, the latter case represents a decentralized problem. In the latter case, the objective function either in the form of per node consumed power or per node achieved throughput is optimized subject to a set of constraints. Similar to the objective function, the set of constraints are only applied to the links using the given node as their transmitting node.

\section{Mobility Experiments}

In this section, we report some of the results of mobility experiments utilizing the integrated schemes of the previous sections. The results are provided for Rician fading environments over a broadband topology. We note that we are solving our QoS-constrained throughput and power optimization problems in broadband ad-hoc networks accommodating space-time 
coded mobile nodes. We consider two cases in which a mobile node is either equipped with one or two antennas. In the case of a double transmit antenna mobile node, we assume that two signals are transmitted simultaneously from the two antennas at each time slot using one of the two MIMO coding schemes STBC or orthogonal STTC. In addition, we assume that the fast fading wireless channel is characterized by a Rician distribution with parameters $\mu=1$ and $\sigma=2$. The channel is assumed to introduce fading factors that are constant over a symbol but vary independently from one symbol to another. We assume the channel state information (CSI) is unknown at the transmitting mobile node and it is known at the receiving mobile node. In the case CSI is known at the transmitting mobile node, the use of beamforming techniques may result in a significant performance enhancement.

We apply our results to an ad-hoc network topology illustrated in Fig. 2. The network parameters are chosen such that the network simulates the communication of four broadband nodes levergaing LOS communication paths. The network consists of mobile nodes $A, B, C$, and $D$, along with 4 links $\mathcal{L}_{1}$ through $\mathcal{L}_{4}$. Each node can be equipped with a single or double antennas. Further, it is able to transmit and/or a receive on multiple links given it cannot transmit and receive at the same time. In the case of simultaneously transmitting on multiple links, the transmission power is split on the outgoing links proportionally.

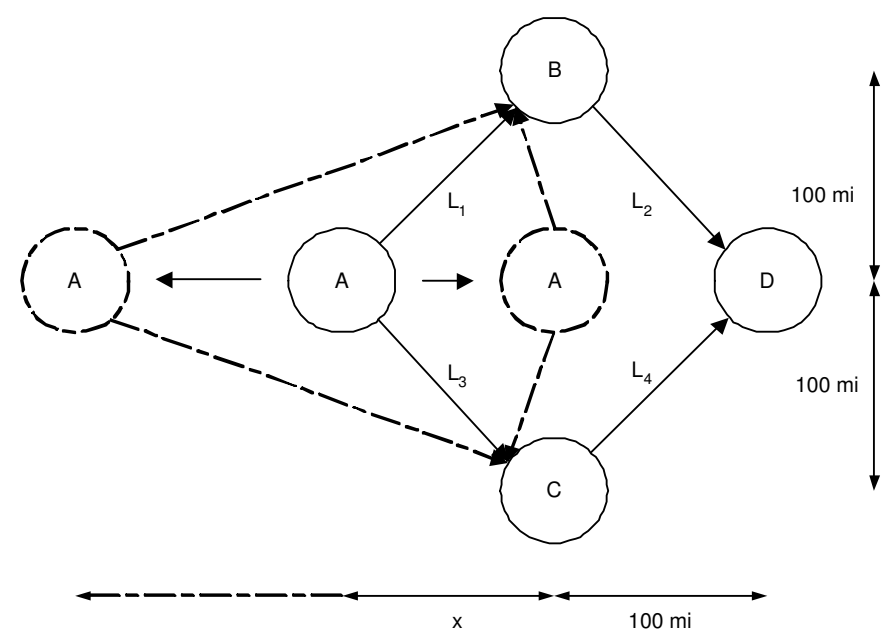

Fig. 2. A sample network topology used in the simulation task.

Originally, nodes $A$ and $D$ are separated by a distance of 100 miles, and so are $B$ and $C$. We allow node $A$ to move across the horizontal axis both toward and away from node $D$. The position of node $A$ from a reference point is indicated by $x$. We select the reference point to be the middle of diagonal line connecting nodes $B$ and $C$. We consider four different scenarios: (1) all of the mobile nodes are single antenna nodes, (2) node $A$ has double antennas utilizing STBCs and the rest of the nodes have a single antenna, (3) node $D$ has double antennas utilizing MRC and the rest of the nodes have a single antenna, and (4) node $A$ has double antennas utilizing orthogonal STTCs and the rest of the nodes have a single antenna. We also make note of our choice of the Trellis of Fig. 1 along with $P P=1$ in scenario (4). In our simulations, each link has a minimum and a maximum transmission power of $0.1 \mathrm{~W}$ and $4 \mathrm{~W}$, respectively. Further, the average power of the noise is assumed to be $1 \mu \mathrm{W}$. We note that the average power of noise can potentially capture the effects of signal interference from other active nodes in the vicinity of a given node and not shown in the topology. However, we assume the minimal existence of such effects in our experiments. All nodes are using BPSK modulation. The baseband bandwidth for each link is $\frac{1}{T}=2 \mathrm{GHz}$ with $\epsilon=2.5 \times 10^{-4}$ and the minimum guaranteed throughput for each link is $128 \mathrm{Kbps}$. A maximum allowable block-loss probability of $10^{-6}$ is set on each link. With the exception of $G_{12}$ and $G_{34}$, the gains for each link are computed as $G_{i i}=\frac{1}{d_{i i}^{2}}$ and $G_{i j}=\frac{\eta}{d_{i j}^{2}}$ for $i \neq j$ where $d_{i j}$ represents propagation path length from the transmitter of link $j$ to the receiver of link $i$. The factor $\eta$ is set as $\eta=0.005$ in our simulations. The gains for $G_{12}$ and $G_{34}$ are set to 0 since we assume a node cannot transmit and receive at the same time. This gives the following gain matrix in terms of $x$.

$$
G=\left[\begin{array}{llll}
1 /\left(x^{2}+10^{4}\right) & 0 & \eta /\left(x^{2}+10^{4}\right) & \eta / 200^{2} \\
\eta /(x+100)^{2} & 1 / 20000 & \eta /(x+100)^{2} & \eta / 200^{2} \\
\eta /\left(x^{2}+10^{4}\right) & \eta / 200^{2} & 1 /\left(x^{2}+10^{4}\right) & 0 \\
\eta /(x+100)^{2} & \eta / 20000 & \eta /(x+100)^{2} & 1 / 20000
\end{array}\right]
$$

BPSK modulation and an RS coder with a block size of 31 is used by all of the transmitters. Working with the 2-state Markov chain fading model in the case of a $1 \times 1$ link with average channel burst lengths of 8 , we calculate the threshold vector as $\left[\begin{array}{lll}0 & 1 & 40\end{array}\right]$. In order to comply with the notion of fairness, we keep the partitioning thresholds the same in the case of other antenna configurations.

Fig. 3 shows the curves of optimal total throughput versus $x$ the position of mobile node $A$ for the different antenna scenarios described above. The curves show that the throughputs are

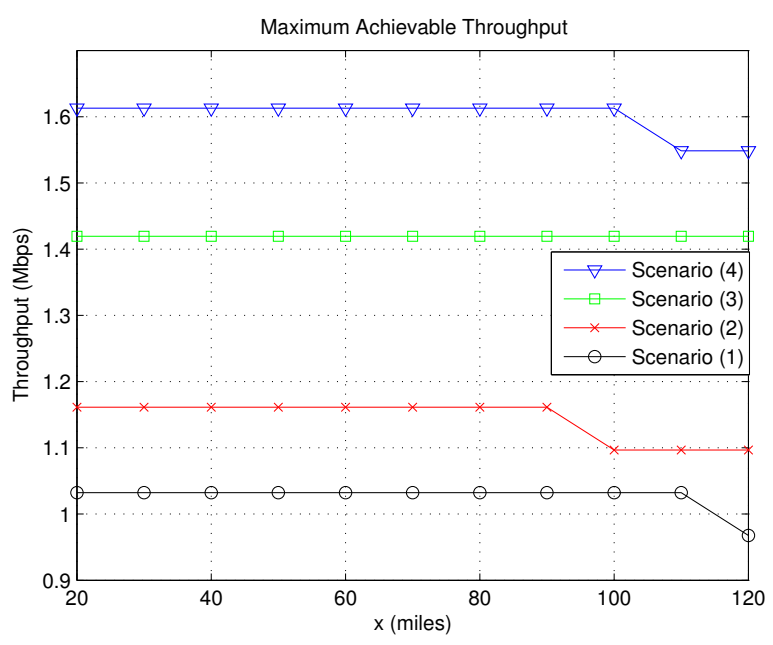

Fig. 3. Optimal curves of total achievable throughput versus $x$.

maintained flat in the range of interest $x \in[20,90]$ miles. As $x$ increases beyond 90 miles, the throughput is decreased due to the loss of signal strength. The most striking observation when comparing the results of individual figures is the fact that the total optimal throughput of scenario (1) is consistently lower than that of the rest. For a given transmission power, we expect 
TABLE I

THE RANGES OF THE OPTIMAL VALUES OF $r_{i}^{*}$ OVER DIFFERENT LINKS FOR DIFFERENT SCENARIOS.

\begin{tabular}{|l|l|l|l|}
\hline & Scenario & $L_{1} / L_{3}$ & $L_{2} / L_{4}$ \\
\hline \hline \multirow{3}{*}{ Rate } & $(1)$ & {$[15 / 31,17 / 31]$} & {$[15 / 31,17 / 31]$} \\
\cline { 2 - 4 } & $(2)$ & {$[23 / 31,25 / 31]$} & {$[19 / 31,21 / 31]$} \\
\cline { 2 - 4 } & $3)$ & {$[23 / 31,25 / 31]$} & {$[19 / 31,21 / 31]$} \\
\cline { 2 - 4 } & $(4)$ & {$[21 / 31,25 / 31]$} & {$[25 / 31,27 / 31]$} \\
\hline \hline \multirow{3}{*}{ Power } & $(1)$ & {$[8 / 31,9 / 31]$} & {$[8 / 31,9 / 31]$} \\
\cline { 2 - 4 } & $(2)$ & {$[8 / 31,9 / 31]$} & {$[8 / 31,9 / 31]$} \\
\cline { 2 - 4 } & $(3)$ & {$[8 / 31,10 / 31]$} & {$[8 / 31,10 / 31]$} \\
\cline { 2 - 4 } & $(4)$ & {$[8 / 31,10 / 31]$} & {$[8 / 31,10 / 31]$} \\
\hline
\end{tabular}

to see a higher throughput when using more antennas. Comparing the throughput of scenario (2) with that of scenario (3), we observe that the former introduces a lower optimal throughput due to the fact that for a fixed $S I N R$ the power efficiency of the former scheme suffers a $3 \mathrm{~dB}$ loss compared to that of the latter scheme for the same transmission power. While the throughput of scenario (4) is the best, it has to be noted that it has the highest complexity of decoding.

Next, we utilize the topology to validate our power minimization approach. Fig. 4 shows the curves of optimal total power versus $x$ the position of mobile node $A$. Again the observations

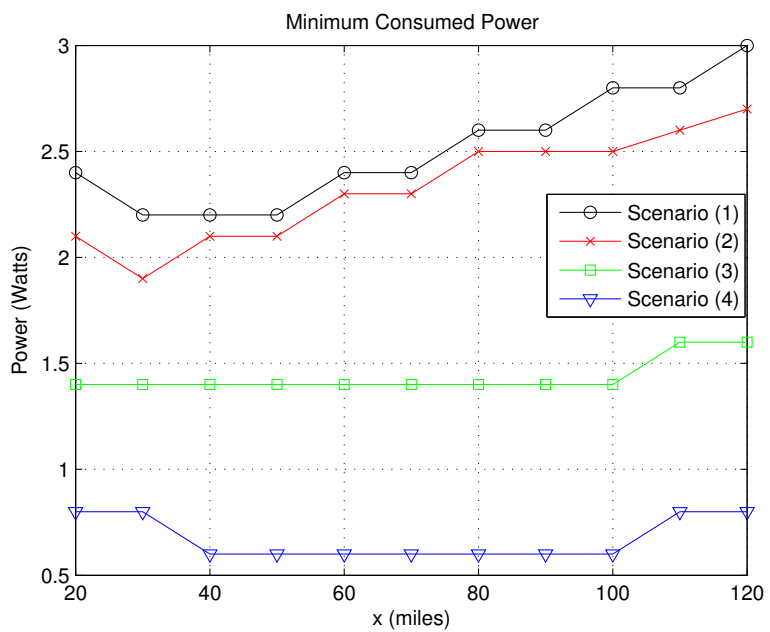

Fig. 4. Optimal curves of total consumed power versus $x$.

are consistent with those of Fig. 3. However, the curves depicted in the figure are now convex. The curves show that total consumed powers are at their minimum levels when the value of $x$ representing the position of node $A$ is in the middle of the range of interest.

Table I illustrates the range of optimal values of $r_{i}^{*}$ over different links for the antenna scenarios described above.

We have observed that utilizing a 3 -state Markov chain lowers the total consumed power compared to the case of a 2-state Markov chain, at the cost of a higher overhead of calculation.

At the end of this section, a discussion of practicality is in order. We note that addressing the tradeoff between practicality and optimality is important as implementing a centralized resource allocation scheme may become overhead prohibitive in a large size network. In order to address the practicality aspect, we note that the formulation of our problem can be applied on a per node basis with a sub-topology of links attached to the node rather than an overall topology. We argue that such a scheme provides a practical alternative to a centralized scheme at the cost of sub-optimality. We refer the reader to [16] for further details.

\section{CONCLUSION}

In this paper, we examined the effects of utilizing MIMO mobile nodes on decreasing total power consumption and/or increasing the achievable throughput in LOS fading ad-hoc networks. We investigated the use of Maximum Ratio Combining (MRC), Space-Time Block Codes (STBCs), and orthogonal Space-Time Trellis Codes (STTCs). We utilized a Rician fading model to represent an LOS operating environment. Relying on the modeling of fading channels with finite-state Markov chains, and the use of Reed-Solomon channel coding, we formulated and solved a pair of constrained resource allocation problems. We also validated our results for a simple yet practical diamond topology under mobility over the range of [20, 120] miles. Our future research is focused on accommodating directional antennas by means of beamforming techniques.

\section{REFERENCES}

[1] S.M. Alamouti, "A Simple Transmitter Diversity Scheme for Wireless Communications," IEEE JSAC, November 1998.

[2] M. Andersin, Z. Rosberg, J. Zander, "Gradual Removals in Cellular PCS with Constrained Power Control and Noise," IEEE/ACM Trans. Networking, April 1997.

[3] N. Bambos, S. Chen, G. Pottie, "Radio Link Admission Algorithms for Wireless Networks with Power Control and Active Link Quality Protection," In Proc. IEEE INFOCOM, 1995.

[4] M. Chiang, D. O'Neil, D. Julian, S. Boyd, "Resource Allocation for QoS Provisioning in Wireless Ad Hoc Networks," In Proc. IEEE GLOBECOM, 2001

[5] S.T. Chung, A. J. Goldsmith, "Degrees of Freedom in Adaptive Modulation: A Unified View," IEEE Trans. on Communications, September 2001.

[6] M. Hayajneh, C.T. Abdallah "Performance of Game Theoretic Power Control Algorithms for Wireless Data in Fading Channels," In Proc. IEEE GLOBECOM, 2003.

[7] A. Papoulis, S.U. Pillai, "Probability, Random Variables, and Stochastic Processes, Fourth Edition,” McGraw-Hill, ISBN 0071122567, 2002.

[8] R. Ramanathan, R. Rosales-Hain, "Topology Control of Multihop Wireless Networks Using Transmit Power Adjustment," In Proc. IEEE INFOCOM, 2000.

[9] M.J. Shah, P.G. Flikkema, "Power-Based Leader Selection in Ad-Hoc Wireless Networks," IEEE Int. Performance, Computing and Communications Conf., 1999.

[10] M.K. Simon, M.S. Alouini, "Digital Communication over Fading Channels: A Unified Approach to Performance Analysis," John Wiley, ISBN 0471317799, 2000.

[11] M.K. Simon, H. Jafarkhani, "Performance Evaluation of SuperOrthogonal Sace-Time Trellis Codes Using a Moment Generating Function-Based Approach," IEEE Trans. on Signal Processing, September 2003.

[12] V. Tarokh, H. Jafarkhani, A.R. Calderbank, "Space-Time Block Coding for Wireless Communications: Performance Results,” IEEE JSAC, March 1999.

[13] V. Tarokh, H. Jafarkhani, A.R. Calderbank, "Space-Time Block Coding from Orthogonal Designs," IEEE Trans. on Information Theory, July 1999.

[14] S. Ulukus, R. Yates, "Adaptive Power Control and MMSE Interference Suppression," ACM/Baltzer Wireless Networks, 1998.

[15] L. Zheng, H. Yousefi'zadeh, H. Jafarkhani, "Resource Allocation in Fading Wireless Ad-Hoc Networks with Temporally Correlated Loss," In Proc. IEEE WCNC, 2004.

[16] H. Yousefi'zadeh, L. Zheng, H. Jafarkhani, "Rate Constrained Power Control in Space-Time Coded Fading Ad-Hoc Networks," In Proc. IEEE GLOBECOM, 2004. 